\title{
Erratum
}

Bull Volcanol (1987) 49:415-434

\section{Changes in magma composition at Arenal volcano, Costa Rica, 1965-1985: Real-time monitoring of open-system differentiation}

Mark K Reagan, James B Gill, Eduardo Malavassi, and Michael O Garcia

In the above article an error was made in Equation (10). The correct version of Equation (10) should be:

$$
\Sigma_{m}=\frac{\left(1-\frac{\mathrm{C}_{\mathrm{o}} \mathrm{F}^{-z}}{\mathrm{C}_{\mathrm{m}}}\right)\left(\mathrm{xC}_{\mathrm{a}} \Sigma_{\mathrm{a}}+\mathrm{yC}_{\mathrm{i}} \Sigma_{\mathrm{i}}\right)}{\mathrm{xC}_{\mathrm{a}}+\mathrm{yC}_{\mathrm{i}}}+\frac{\mathrm{C}_{\mathrm{o}} \Sigma_{\mathrm{o}} \mathrm{F}^{-z}}{\mathrm{C}_{\mathrm{m}}}
$$

